\title{
Decoding natural astrocyte rhythms: dynamic actin waves result from environmental sensing by primary rodent astrocytes
}

\author{
Kate M. O’Neill ${ }^{1}+$, Emanuela Saracino ${ }^{2}+$ Barbara Barile $^{3}$, Nicholas J. Mennona ${ }^{1,4}$, \\ Maria Grazia Mola ${ }^{3}$, Spandan Pathak ${ }^{1}$, Tamara Posati ${ }^{2}$, Roberto Zamboni ${ }^{2}$, Grazia P. Nicchia ${ }^{3}$, \\ Valentina Benfenati ${ }^{2 *}$, Wolfgang Losert ${ }^{1,4^{*}}$ \\ ${ }^{1}$ Inst. for Physical Science and Technology, Univ. of Maryland, College Park, MD, USA \\ ${ }^{2}$ Inst. of Organic Synthesis and Photoreactivity, National Research Council of Italy, Bologna, Italy \\ ${ }^{3}$ Dept. of Bioscience, Biotechnology and Biopharmaceutics, Univ. of Bari Aldo Moro, Bari, Italy \\ ${ }^{4}$ Physics Dept., Univ. of Maryland, College Park, MD, USA \\ t equal contributions as co-first authors \\ *valentina.benfenati@isof.cnr.it andwlosert@umd.edu (equal contributions as \\ co-corresponding authors)
}

\begin{abstract}
$\underline{\text { Abstract }}$
Astrocytes are key regulators of brain homeostasis, which is essential for proper cognitive function. The role of cytoskeletal dynamics in this critical regulatory process is unknown. Here we find that actin is dynamic in certain subcellular regions, especially near the cell boundary. Our results further indicate that actin dynamics concentrates into "hotspot" regions that selectively respond to certain chemophysical stimuli, specifically the homeostatic challenges of ion or water concentration increases. Substrate topography makes actin dynamics more frequent yet weaker, and it also alters actin network structure. Superresolution images analyzed with a filament extraction algorithm demonstrate that surface topography is associated with a predominant perpendicular alignment of actin filaments near the cell boundary whereas flat substrates result in an actin cortex mainly parallel to the cell boundary. Thus, actin structure and dynamics together integrate information from different aspects of the environment that might steer the operation of neural cell networks.
\end{abstract}

\section{Short Title}

Homeostatic actin dynamics in astrocytes

\section{$\underline{\text { Teaser }}$}

Astrocytes display dynamic actin that is modulated by combinations of chemophysical stimuli and environmental topographies. 


\section{Introduction}

The ability of the brain to receive and compute information depends on the unique abilities of its component brain cells. In particular, astrocytes play crucial roles in cognitive function by maintaining electrochemical gradients of ions and both responding to and counteracting changes in the chemophysical environment of the extracellular milieu [1-3]. More recently, the importance of astrocytes' structural and functional properties has been highlighted for organ- and system-level functions, including synaptic plasticity, memory formation, learning, and regulation of sleep and metabolic activity $[4,5]$.

Astrocytes exhibit a branched morphology [1, 3] and express a variety of transmembrane proteins and receptors in a specific, patchy distribution throughout the cell, resulting in so-called functional microdomains. This molecular and structural patchwork allows astrocytes to uptake and pump ions, osmotically drive water and neurotransmitters across the cell, and redistribute and spatially "buffer" solute concentrations [6]. In this way, astrocytes precisely control the extracellular environment locally by continuously monitoring, sensing, and balancing changes in response to the neuronal network activity. At the same time, the gap junction coupling between astrocytes serves as a signaling path enabling synchronicity and lateral communication within the larger astroglia syncytia [7].

Though gap junctional communication coupling enables dynamic exchange at the ionic and molecular scales $[8,9]$, questions remain how these dynamics couple with, modulate, and impact responses of cell networks or of the whole brain. Here we investigate the actin cytoskeleton as an integrator from the molecular to the systems scale due to its ability to self-assemble into dynamic networks.

Indeed, experiments on fixed samples indicate that the astrocytic actin cytoskeleton plays a role in several homeostatic processes, such as volume regulation and potassium spatial buffering [1012]. However, much less is known about the dynamics of the actin cytoskeleton in these cellular processes. For migrating cells - such as neutrophils, metastatic cancer cells, and even microglia - actin dynamics has been mostly considered as a facilitator of changes in cell morphology. For example, a recent study in $\mathrm{T}$ cells demonstrated the importance of global (whole cell) actin dynamics in facilitating immunological synapse formation [13].

Moreover, recent studies across multiple cell types have demonstrated not only that the actin cytoskeleton is dynamic when a cell changes shape and moves but also that the actin cytoskeleton is an excitable system with its own intrinsic dynamics, including sustained waves and oscillations that persist independently of changes to cell shape [14-21]. The source of actin dynamics is thought to be twofold: i) a cytoskeletal excitable network of proteins (CEN) that drives cycles of polymerization and depolymerization typically lasting a few seconds, and ii) a signal transduction excitable network (STEN) that can self-organize these local oscillations into larger scale waves that drive cell migration $[17,18,22,23]$.

Indeed, CEN waves can carry information. Actin waves have been shown to be sensitive to extracellular local topography upon which cells are cultured [24, 25] and are important for biological function [22, 26], such as surveillance of the brain parenchyma by microglia [27]. 
These waves are due to the second scale polymerization and depolymerization dynamics that occur locally due to CEN and are usually obscured by the longer timescale wave dynamics, but they can be revealed by suppression of STEN $[17,18,22,23]$.

We propose that, in astrocytes, CEN dynamics dominate, thus leading to local actin dynamics that are stationary in space and occur on a second timescale. Thus, cytoskeletal excitable networks carry information about homeostatic challenges that occur at the nanometer, ionic, and molecular scales. The actin cytoskeleton therefore serves as the bridge from these smaller scales to larger scales and facilitates the global homeostatic response of astrocytes.

To evaluate our hypothesis, we use optical flow algorithms and shape analysis on images of primary astrocytes transduced with actin-GFP. We demonstrate that actin dynamics occurs in astrocytes and that it can be triggered by extracellular environment modifications, such as an increase in ion concentrations or a change in nanomechanical features. The spatial distribution of the actin dynamics is localized near the boundary of the cell and is concentrated into patches we term "hotspots".

Notably, the strength of the actin dynamics is different depending on whether the exposure is to an increase in extracellular potassium or to hypotonic challenge (referred to as "triggering" conditions and abbreviated as "high $\mathrm{K}+$ " and "hypotonic", respectively). In addition, activity identified in the processes of differentiated astrocytes, induced by nanotopography, is weaker but more frequent when compared to undifferentiated cells. Superresolution imaging by STimulated Emission Depletion (STED) microscopy and analysis with a filament extraction algorithm demonstrates that the specific orientation of actin filament structures occurs only in differentiated cells, indicating that both structure and dynamics carry information about the environment.

Overall, our results indicate that actin is dynamic in astrocytes and behaves collectively by clustering into "hotspot" regions that respond in distinct ways to different stimuli and may drive the homeostatic response of the neural cell network.

\section{$\underline{\text { Results }}$}

\section{Primary astrocytes display actin dynamics in response to chemical cues}

Our live imaging results demonstrate that astrocytes consistently display actin dynamics, particularly near the boundary of the cell. Representative overlays of fluorescent images are shown in Figure 1A (A1, inset A3 for representative control timelapse and A2, inset A4 for representative "triggering" timelapse). In this representation, a perfectly stationary cell would appear all white; the appearance of different colors indicates non-overlapping structures at either earlier (more blue) or later (more red) times. To quantify actin dynamics, we employ an optical flow method [28] similar to that used in our previous work [19]. We additionally use shape analysis [16, 29, 30] to separately consider actin dynamics within the whole cell, boundary region, and bulk region (see Figure 1B and 1C for schematics and Materials and Methods for 133 more detail). 
To obtain an overall picture of how actin dynamics changes between control and triggering conditions, we first examined the strength of actin dynamics within specific regions (Figure 1C2). Both triggering conditions show increased strength of actin dynamics within the boundary region ( $\mathrm{p}=0.014$ and $\mathrm{p}=0.028$ for high $\mathrm{K}+$ and hypotonic, respectively) but not within the bulk region ( $\mathrm{p}=0.050$ and $\mathrm{p}=0.057$ for high $\mathrm{K}+$ and hypotonic, respectively) when compared to paired controls ( $\mathrm{p}$-values determined by paired t-tests).

We then wanted to understand whether actin dynamics becomes relatively more probable in the boundary region and/or in the bulk region after a triggering stimulus. To accomplish this, we calculate a relative fraction and find a significant shift in actin dynamics towards the boundary under high $\mathrm{K}+$ conditions compared to paired controls; there is no significant difference for hypotonic conditions compared to paired controls (Figure 1C3; $\mathrm{p}=0.026$ and $\mathrm{p}=0.466$, respectively, as determined by paired t-test).

To delve into distance dependence of actin dynamics more closely, we quantify the distance from instances of actin dynamics to the cell boundary (true boundary shown in Figure 1B). Curves of the representative cells shown in Figure 1A are shown in Figure 1D1 with the boundary region and bulk region labeled. Since we are most interested in the high actin activity near the boundary, we compare how the probability of actin dynamics changes over distance when comparing the two triggering conditions to their matched controls. High $\mathrm{K}+$ cells are significantly less likely to show actin dynamics near the boundary edge compared to their matched controls, though the mean difference is extremely small $(\mathrm{p}=0.019$ for $3.24 \mu \mathrm{m}$ and $\mathrm{p}=0.021$ for $3.6 \mu \mathrm{m}$, determined by repeated measures ANOVA). On the other hand, hypotonic cells are significantly more likely to show actin dynamics within the boundary region compared to matched controls, specifically between 0.36 and $2.52 \mu \mathrm{m}$ from the boundary $(\mathrm{p}<0.05$ for all distances as determined by repeated measures ANOVA; refer to Figure 1D3 legend for exact pvalues). Taken together with the data from Figure 1C3, we show that a higher probability of actin dynamics at specific distances is not necessarily accompanied by an overall shift to the boundary region.

These results indicate the following: i) actin dynamics has a higher probability of occurring under high $\mathrm{K}+$ conditions (Figure 1C2), ii) a relative shift of actin dynamics to the boundary is observed when cells are exposed to high $\mathrm{K}+$ (Figure 1C3), iii) increased probability of actin dynamics at specific distances is observed when cells are exposed to hypotonic challenge (Figure 1D3), and iv) for both triggering conditions, the strength of actin dynamics increases within the boundary region but not within the bulk region (Figure 1C2).

\section{Actin dynamics within astrocytes is clustered into active "hotspot" regions}

While characterizing actin dynamics within astrocytes, we noticed that many cells seemed to have active regions near the boundary (Figure 2A1) that we suspected were responsible for most of the actin dynamics. To identify these "hotspot" regions, we applied the image processing techniques of erosion and dilation to identify areas of persistent activity (Figure 2A2). After applying thresholds for activity magnitude and probability and hotspot size, we segment regions that meet these criteria (Figure 2A3, inset Figure 2A4). 
We first compared the strength of actin dynamics within the hotspot regions, considering the boundary and bulk regions separately as in Figure 1C2. Following the trend of activity within the whole cell, we found that actin dynamics is stronger in cells exposed to both high $\mathrm{K}+$ and hypotonic, specifically within the boundary region $(\mathrm{p}=0.015$ and $\mathrm{p}=0.032$, respectively, as determined by paired $t$-test) and not within the bulk region ( $p=0.464$ and $p=0.527$, respectively, as determined by paired t-test) as shown in Figure 2B1. We also investigated the relative strength of hotspot dynamics where the average magnitude within hotspots is normalized by the average magnitude of dynamics within the whole cell (Figure 2B2). When comparing this relative strength between cells in triggering conditions and their matched controls, we found that only high $\mathrm{K}+$ cells displayed significantly stronger actin dynamics within hotspot regions compared to the whole cell ( $\mathrm{p}=0.028$ for control vs. $\mathrm{K}+$ and $\mathrm{p}=0.087$ for control vs. hypotonic as determined by paired t-test). Finally, we investigated whether actin dynamics in these hotspot regions is more persistent and found that the duration is not significantly different for either high $\mathrm{K}+$ cells or hypotonic cells compared to matched controls $(\mathrm{p}=0.095$ and $\mathrm{p}=0.831$, respectively, as determined by paired t-test), as shown in Figure 2 B3.

To provide a static visualization of actin dynamics within these hotspots, we show traditional actin fluorescence kymographs (Figure 2C1) and novel actin dynamics kymographs (Figure 2C2) for the three largest hotspot regions (reg, orange, and yellow) in the representative cell. Both types of kymographs clearly show stronger dynamics in the triggering condition. Taken together, these results show that hotspot regions respond distinctly to different stimuli and that they are particularly active in general and specifically reactive to the high $\mathrm{K}+$ chemical stimulus.

\section{$\underline{\text { Astrocytes respond to nanotopographic surfaces with distinct actin dynamics }}$}

Finally, we took advantage of the topographic features in hydrotalcyte-like compound (HTlc) films to understand how actin dynamics is altered by mechanical stimuli at the nanometer scale. HTlc films direct polygonal astrocytes to develop a highly branched and differentiated morphology, thus recapitulating in in vitro astrocytes the functional microdomains regulating water and potassium homeostasis that would be seen in vivo [31,32].

To this end, we cultured primary rat astrocytes on PDL-coated glass or on HTlc nanoparticle films and imaged them under control conditions using the same parameters as before. Morphological and functional differences between cells grown on these two surfaces have been thoroughly characterized previously [31], and similar differences are observed in our system as shown in Figure 3A.

We first compared how actin dynamics differs in the various regions of PDL cells versus HTlc cells. We found that PDL cells show significantly stronger dynamics both within the boundary region and within the bulk region (Figure 3B1, p $<0.001$ as determined by unpaired t-test). Surprisingly though, when comparing boundary to bulk within each nanotopographic surface (Figure 3B2), we found that both PDL and HTlc show significantly stronger boundary dynamics compared to bulk dynamics $(\mathrm{p}<0.001$ for PDL and $\mathrm{p}=0.021$ for HTlc as determined by paired $t-$ test). 
We next compared how likely cells on the different surfaces are to show actin dynamics. When comparing within each region, we found that HTlc cells are significantly more likely to have actin dynamics, both within the boundary region and within the bulk region compared to PDL cells (Figure 2C1; $\mathrm{p}=0.014$ and $\mathrm{p}=0.020$, respectively, as determined by unpaired t-test). In this case, there is no significant difference between boundary and bulk for either surface (Figure 2C2; $\mathrm{p}=0.072$ for PDL and $\mathrm{p}=0.184$ for HTlc as determined by paired $\mathrm{t}$-test).

Finally, we evaluated distance dependence in Figure 3D similar to in Figure 1D. We show the full distance curves in Figure 1D1 for the representative cells. When comparing the individual distances within boundary region, we see that HTlc cells are significantly more likely to have actin dynamics (Figure 3D2; $\mathbf{p}<0.05$ for all distances between 0.36 and $3.6 \mu \mathrm{m}$ as determined by two-way ANOVA). Finally, when normalizing for size of the boundary (Figure 3D3), we do not find any difference in the relative fractions of actin dynamics within the boundary region compared to the bulk region for either surface ( $\mathrm{p}=0.287$ for PDL and $\mathrm{p}=0.058$ for HTlc as determined by paired t-test). Taken together, these results indicate that HTlc cells show more frequent and weaker actin dynamics than PDL cells.

Astrocytes sense nanotopographic cues through actin orientation near the boundary

Inspired by our analysis of actin dynamics in live astrocytes, we sought to understand i) why certain "hotspots" of actin activity are more prevalent in cells grown on PDL than in cells grown on HTlc and ii) whether the characteristics of actin dynamics are related to the underlying actin structure. To this end, we cultured primary rat astrocytes on PDL-coated glass or on HTlc films and performed STED microscopy on fixed cells stained for F-actin and the intermediate filament protein glial fibrillary acid protein (GFAP). Representative images are shown in Figure 4. HTlc preferentially induces stellate morphology characteristic of differentiated astrocytes, whereas astrocytes grown on PDL-coated glass display the polygonal shapes characteristic of undifferentiated astrocytes.

Confocal images of F-actin labeled astrocytes reveal that there is a marked tendency for cells grown on PDL (Figure 4A) to display a non-differentiated and dynamic phenotype with distinct leading and trailing edges and several motile structures (i.e. lamellipodia, filopodia, stress fibers; Figure 4C). Conversely, in the "star" shaped cells grown on HTlc (Figure 4B), the same motile elements that would indicate direction of propagation are not clearly visible (Figure 4D). Moreover, the astrocytic actin is seen to "burst" out of the actin cortex in HTlc cells (Figure 4D). This bursting or "flaring" behavior is indicative of a functional microdomain, known to be present in differentiated astrocytes grown on HTlc [31]. Although many regions in the representative HTlc cell display this microdomain morphology, the actin dynamics of astrocytes plated on HTlc is of lower magnitude than that of astrocytes grown on PDL (refer to Figure 3B). Therefore, we chose to investigate how astrocytic actin senses the different mechanical environments presented by PDL-coated glass and HTlc films and the subsequent cytoskeleton remodeling at a nanoscale level via superresolution imaging. We used a method for quantitative, semi-automated analysis of the actin bundles' preferential orientation [33] to assess the involvement of actin organization in sensing the local mechanical environment around astrocytes. 
Image analysis was performed by combining segmentation via an anisotropic, rotating Laplacian of gaussian (LoG) kernel with a hierarchical cluster analysis (see Figure 5A for a schematic overview and Materials and Methods for a detailed description). These image processing methods allow for the analysis of actin angle organization and the determination of fractions of actin roughly parallel versus roughly perpendicular to the leading edge of the cell boundary (Figure 5B). From our clustered data, we determine that PDL astrocytic actin is oriented more parallel relative to the leading edge of the astrocyte, whereas HTlc astrocytic actin is oriented more perpendicular (Figure 5C). The difference seen between the actin of cells grown on PDL versus on HTlc is significant as the cluster algorithm identifies two clusters within the data. Moreover, we see the cluster groups largely correspond with the chosen nanotopography, with an error of seven out of 37 cells: one HTlc cell is misclustered, and six PDL cells are misclustered (i.e. six PDL cells can be found in the cluster of predicted HTlc actin fractions). While the differences observed between astrocytes grown on HTlc and those grown on PDL at a dynamic, micron scale do not necessarily correspond to the differences seen in structure at the nanometer scale, our analytical approach reveals that the underlying nanoscale organization of actin within astrocytes does indeed change when the cells are exposed to different nanotopographic surfaces.

\section{$\underline{\text { Discussion }}$}

We show for the first time, by means of live timelapse confocal microscopy, that astrocytes respond to changes in the chemophysical environment with changes in actin dynamics. Actin is spatiotemporally organized into micron scale territories that enhance the dynamics in response to chemophysical stresses. Topography enhances the frequency of these dynamics but lowers their strength.

Our study reveals that actin dynamics occurs persistently and on different spatial scales, with local actin dynamics detectable near the boundary even when the boundary itself does not shift measurably. Dynamics are stronger in cells grown on PDL and cluster into hotspot regions, whereas they are more frequent in cells grown on HTlc and do not cluster into large enough regions to be detected with confocal microscopy. Both high potassium and hypotonic stimuli increase the strength of actin dynamics within the boundary region, but high potassium preferentially increases the strength of dynamics within hotspots, which is likely why the relative fraction of dynamics shifts towards the boundary. We find that these dynamics are localized to one or multiple boundary regions characterized by a lack of strong stress fibers, with a spatial scale larger than individual synapses.

While astrocytes do not change shape substantially on the short timescales of our experiments, we see small filopodial and lamellopodial structures near the cell boundary that appear to "idle" by rapidly extending and retracting, effectively sampling their local environment. It is in these regions that we most often observe actin dynamics.

Similar to previous studies, we note that on longer timescales astrocytes are extremely plastic cells. For example, after injury, astrocytes in vivo become reactive and highly ramified [34, 35]. Astrocytes cultured in vitro, however, appear polygonal and lose their starlike shape when the McCarthy and de Vellis preparation method is used [36-38], but it is possible to induce 
differentiation and in vivo-like structural and functional properties over the course of a few days using a previously validated approach [31, 32], which consists of growing astrocytes on HTlc nanostructured films.

Surprisingly, actin is more dynamic in polygonal cells grown on flat surfaces (glass coverslips coated with PDL), which also favors the formation "hotspots" of actin dynamics compared to highly differentiated cells grown on nanostructured surfaces (HTlc films). The frequency of activity and the structure of the network is distinct for differentiated cells grown on HTlc, with filaments preferentially oriented perpendicular to the cell boundary.

The presence of a dynamic cytoskeleton during structural remodeling in astrocytes has been hypothesized to support the rapid morphological changes that occur in perisynaptic astrocytes processes (PAPs), seen in organotypic culture, in response to synaptic activity and long-termpotentiation [39]. Our results support this hypothesis, but, unexpectedly, we found that the strength of actin dynamics is lowered in differentiated astrocytes with respect to polygonal cells while the frequency of the movements is higher. Dynamic hotspots in differentiated cells might present as miniature versions of lamellipodia and filopodia, the F-actin-rich subcellular compartments of migrating non-neuronal cells [40].

Our STED data might also provide the key to understanding the conclusion that nondifferentiated cells perform more robust cytoskeleton dynamics: a more parallel and dispersed organization of actin fibers into a meshwork underneath the cell membrane is likely to confer mechanical plasticity to the plasma membrane when compared to more differentiated cells on HTLc, where actin fibers are packed into "actin rails" and not only maintain the cell shape (especially in the cellular processes) but also might cause membrane stiffness or resistance to motility. We also hypothesize that this distinct structure will lead to differences in the interaction with neighboring cells. As stellation enables communication with other cell types, perpendicular actin may ensure processes are directed along a specific line of communication. Our finding that almost all cells on HTlc have actin structures perpendicular to the boundary affirms the ability of HTlc to encourage differentiation of astrocytes [31]. This analysis also hints that some cells grown on PDL have actin structures that "look" like the actin structures of stellated cells grown on HTlc, consistent with previous observations that growth of astrocytes on PDL delays but does not fully suppress spontaneous or gliotic differentiation that might occur in standard cell culture $[11,31,41,42]$.

Our previous studies demonstrated that astrocytes grown on HTlc are more efficient in their homeostatic process. In particular, the expression and function of Kir4.1 and AQP4 as well as the functional response to anisotonic challenge are improved in differentiated cells [31, 32]. In this respect, a faster and prompter response to chemophysical challenges might not require a major effort from the dynamic actin network, which might explain the lower magnitude of actin dynamics in differentiated cells. Moreover, the functionally better equipped differentiated astrocytes may also be able to more easily sense changes in the extracellular environment and react more frequently to them via actin dynamics. Thus, our findings are no longer surprising if we consider that astrocytes harness the excitable dynamics of actin to codify and convey information coming from the extracellular milieu. 
Our finding that actin dynamics is altered by chemophysical stimuli and topography raises important questions for astrocyte physiology and pathophysiology: Is it possible to control and/or modulate the size of the spatial region where actin dynamically rearranges by activating a Signal Transduction Excitable Network (STEN)? Studies on other cell types suggest that activating STEN would enable traveling waves of varying sizes and may even trigger cell migration [17, $18,22]$. Could extreme conditions strengthen and alter the actin dynamics so much as to drive astrocyte differentiation? These questions remain to be elucidated, and studies in microglia [27] - which demonstrated that actin dynamics is critical for their principal function, surveillance indicate that actin dynamics also plays an important role in vivo in neural cells. We propose that actin dynamics in astrocytes is critical for their principal functions: maintaining homeostasis and modulating neuronal communication. Finally, given that cytoskeletal alterations are reported in a variety of neurological conditions characterized by a gliotic state [43], we expect investigations of cytoskeletal dynamics in defined animal models to be an important area of exploration to validate the pathophysiological relevance [44] of our findings.

\section{$\underline{\text { Materials and Methods }}$}

$\underline{\text { Rat cortical astrocyte culture preparation, maintenance, and plating }}$

\section{Cell culture:}

Primary astrocytes were obtained from Sprague Dawley rats housed at the University of Maryland (in concordance with the recommendations of and approval by the University of Maryland Institutional Animal Care and Use Committee; protocols R-JAN-18-05 and R-FEB-2104) or from Wistar rats housed at the University of Bologna (in concordance with the Italian and European law of protection of laboratory animals and the approval of the local bioethical committee, under the supervision of the veterinary commission for animal care and comfort of the University of Bologna and approved protocol from Italian Ministry of Health; ethical protocol number ID 360/2017 PR, released on May 2017, valid for 5 years).

Primary cultures of astrocytes were prepared as described previously, from newborn rat pups between postnatal days 1 and $2[3,36,45]$. Briefly, neonatal cerebral cortices devoid of meninges were gently triturated, filtered with a $70 \mu \mathrm{m}$ cell strainer (cat. no. 22-363-548; Fisher Scientific, MA, USA), and plated in T25 cell culture flasks (cat. no. 229331; CELLTREAT Scientific Products, MA, USA) containing Dulbecco's Modified Eagle Medium (DMEM) with GlutaMAX and high glucose (cat. no. 10-566-024; Fisher Scientific, MA, USA) supplemented with 15\% fetal bovine serum (FBS; cat. no. 100-106; Gemini Bio-Products, CA, USA) and penicillin-streptomycin at $100 \mathrm{U} / \mathrm{mL}$ and $100 \mu \mathrm{g} / \mathrm{mL}$, respectively (cat. no. 15070063; Thermo Fisher Scientific, MA, USA). Flasks were maintained in an incubator at $37{ }^{\circ} \mathrm{C}, 5 \% \mathrm{CO}_{2}$, and proper humidity levels for two weeks. During this period, we replaced cell medium every two days, and flasks were gently shaken when necessary to remove undesired microglial cells. When confluence was reached, astrocytes were dispersed using trypsin-EDTA $0.25 \%$ (cat. no. 25200056, Thermo Fisher Scientific, MA, USA), and the cell suspension was re-plated on glass coverslips functionalized with Poly-D-lysine hydrobromide (PDL; cat. no. P7886; Sigma-Aldrich, MO, USA) as previously described [31, 45-48] or with HTlc as previously 
described $[31,49]$. Cells were plated at a density of $5-10 \times 10^{3}$ per dish and maintained in culture medium containing $10 \%$ FBS.

\section{PDL and HTlc film preparation:}

Microscope coverslips were functionalized with PDL to serve as a control surface or with Zn-Al hydrotalcite nanoparticles (HTlc) to induce morphological differentiation of astrocytes. For live imaging, PDL was used at a working concentration of $0.1 \mathrm{mg} / \mathrm{mL}$, and cells were cultured for three to seven days after replating prior to imaging. For STED imaging, PDL was used at a working concentration of $0.01 \mathrm{mg} / \mathrm{mL}$, and astrocytes were cultured for five days after replating prior to fixation. In both cases, coverslips were coated with PDL for 20 minutes at room temperature and washed three times with approximately $500 \mu \mathrm{L}$ of sterile $\mathrm{H}_{2} \mathrm{O}$. Coverslips were functionalized with HTlc by adding the HTlc suspension drop by drop on the coverslips and allowed to dry overnight. Once dried, the HTlc coverslips were sterilized by UV for approximately $30 \mathrm{~min}$.

Transduction of cells with actin-GFP

To visualize actin dynamics, astrocytes were transduced with CellLight Actin-GFP, BacMam 2.0 (cat. no. C10506; ThermoFisher Scientific, MA, USA) at a concentration of 100 particles per cell. At $48 \mathrm{hr}$ prior to live imaging, $5-10 \mu \mathrm{L}$ (depending on the plating density) of the reagent was added to the cell culture media. A full media change was performed $16-20 \mathrm{hr}$ after transduction, and imaging was performed $24-48 \mathrm{hr}$ after the full media change.

\section{Live confocal imaging of transduced astrocytes}

Live imaging timelapses were acquired at the University of Maryland CMNS Imaging Core using a PerkinElmer spinning disk confocal microscope with an oil immersion 40x objective $\left(1.30 \mathrm{NA} ; 0.36 \mu \mathrm{m} /\right.$ pixel) and under temperature, $\mathrm{CO}_{2}$, and humidity control. The microscope was equipped with a Hamamatsu ImagEM X2 EM-CCD camera (C9100-23B), which recorded 16 bit images. Data acquisition was performed with PerkinElmer's Volocity software (version 6.4.0). A subset of timelapses were taken at the CNR on an inverted confocal microscope (Crisel Instruments) using a 40x water immersion objective $(0.16 \mu \mathrm{m} / \mathrm{pixel})$.

Transduced astrocytes were identified by a positive signal in the $488 \mathrm{~nm}$ laser channel. After imaging parameters were set, the media was changed from growth media to standard external solution (control) followed by one of the triggering conditions (high potassium solution or hypotonic solution). Single-plane images were acquired every $2 \mathrm{sec}$ in the 488 channel. A consistent z-plane was assured with Perfect Focus (Nikon PFS).

$\underline{\text { Immunostaining and superresolution imaging of fixed astrocytes }}$

\section{Antibodies:}

Mouse monoclonal Anti-Glial Fibrillary Acidic Protein (GFAP) antibody (diluted 1:200; cat. no. G3893; Sigma-Aldrich, MO, USA) and Goat anti-Mouse IgG (H+L) Highly CrossAdsorbed Secondary Antibody, Alexa Fluor 594 (diluted 1:300; cat. no. A-11032; Thermo Fisher Scientific, MA, USA) were used as primary and secondary antibody, respectively, for GFAP immunostaining. Alexa Fluor 488 Phalloidin (diluted 1:500; cat. no. A12379; Thermo Fisher Scientific, MA, USA) was used for direct labelling of actin. 
STED immunostaining:

Immunofluorescence for gated STED (gSTED) was performed as indicated in the Quick Guide to the STED Sample Preparation (www.leica-microsystems.com), with some slight adjustments as previously reported $[50,51]$. Astrocytes were plated on the PDL and HTlc-treated coverslips and cultured for five days. On the fifth day of culture, cells were fixed with $2 \%$ paraformaldehyde (cat. no. P6148; Sigma-Aldrich, MO, USA) in PBS for 10 min, washed with PBS, and permeabilized with $0.3 \%$ Triton X-100 (cat. no. T8787; Sigma-Aldrich, MO, USA) for 10 min. After blocking with $0.1 \%$ gelatin in PBS, fixed astrocytes were incubated with GFAPprimary antibody for $1 \mathrm{hr}$ at room temperature. Cells were then rinsed with $0.1 \%$ gelatin-PBS and co-incubated with Alexa Fluor 488 Phalloidin and Alexa Fluor 594-conjugated secondary antibody for $1 \mathrm{hr}$ at room temperature. After washing with PBS, coverslips were mounted on microscope slides by using the ProLong Glass Antifade Mountant (cat. no. P36980; Thermo Fisher Scientific, MA, USA) without DAPI, as indicated in Leica official guide, and imaged with both confocal and STED microscopy.

\section{STED imaging:}

473 Confocal and STED images of fixed astrocytes grown on PDL and HTlc were acquired using a Leica TCS SP8 3X microscope, provided with AOTF and AOBS, white light laser (WLL), Hybrid Detectors (HyD), and two STED lasers (592 nm, $660 \mathrm{~nm}$ ) [51]. A Leica HC PL APO 100x/1.40 NA Oil STED White objective and Type F Immersion liquid with a refractive index of 1.5 were used. Before starting imaging, the excitation and the doughnut-shaped STED beams were switched on (WLL set laser power $=70 \%$; STED-592 nm set laser power $=98 \%$ ), aligned, and allowed to reach operating temperature. The beam alignment was repeated whenever necessary. Excitation of the Alexa Fluor 488 dye was achieved using a continuous-wave $488 \mathrm{~nm}$ laser line (NKT Photonics supercontinuum laser). For superresolution imaging of actin, g-STED was performed using the continuous wave $592 \mathrm{~nm}$-emitting STED fiber laser. More detailed acquisition settings are reported in Table 1. All confocal and STED images were acquired at a set room temperature of $20^{\circ} \mathrm{C}$ under constant Acquisition Mode settings (Format scanning resolution: 1024x1024 pixels; Scan Speed: $100 \mathrm{~Hz}$ ).

\section{Criteria for acquisitions of ROIs:}

Single or few and well-spaced cells were preferentially chosen for STED imaging to ensure no overlap of actin structure belonging to different cells. Actin and GFAP images of the whole cell were first acquired by confocal microscopy at an original magnification of 100x. Then, multiple random regions of interest (ROIs) $(\sim 20 \times 20 \mu \mathrm{m}$ sized) were selected and imaged using an extra optical zoom (from 5 to 7) by confocal and STED microscopy. Three independent experiments were conducted.

Synthesis of ZnAl-HTlc nanoparticles and film preparation

\section{Colloidal aqueous dispersion of ZnAl-HTlc nanoparticles having the formula} $\left[\mathrm{Zn}_{0.72} \mathrm{Al}_{0.28}(\mathrm{OH})_{2}\right] \mathrm{Br}_{0.28} \quad 0.69 \mathrm{H}_{2} \mathrm{O}$ were prepared by the double-microemulsion technique previously described [31]. For the film preparation, a 125 or $160 \mu \mathrm{L}$ aliquot of ZnAl-HTlc colloidal dispersion at a concentration of $1.2 \mathrm{mg} / \mathrm{mL}$ was dropped onto 15 or $19 \mathrm{~mm}$ diameter glass coverslips and successively dried for $4 \mathrm{hr}$ in a sterile hood. The obtained ZnAl-HTlc films were used to culture astrocytes and as substrates for live dynamics imaging and fixed STED 
imaging as described above. In this manuscript, the ZnAl-HTlc films are referred to simply as HTlc.

\section{$\underline{\text { Solutions and chemicals }}$}

All salts and chemicals employed for the investigations were of the highest purity grade. For live imaging experiments, the standard external solution ("control") was (mM): $140 \mathrm{NaCl}, 4 \mathrm{KCl}$, $2 \mathrm{MgCl}_{2}, 2 \mathrm{CaCl}_{2}, 10$ HEPES, 5 glucose, $\mathrm{pH} 7.4$ with $\mathrm{NaOH}$ and osmolarity adjusted to $\sim 315$ mOsm with mannitol. When using external solutions with different ionic compositions, such as the high potassium solution ("high $\mathrm{K}+$ ", [40 Mm]), salts were replaced equimolarly. The hypotonic extracellular solution ("hypotonic") of $260 \mathrm{mOsm} / \mathrm{L}$ was obtained by omitting mannitol in the control solution. When imaging, media was changed carefully to prevent accidental, mechanical stimulation of cells with the pipette. Moreover, we controlled for any effects that changing media might cause by changing media prior to imaging under control conditions (from growth media to the standard external solution) and under triggering conditions (from the standard external solution to high $\mathrm{K}+$ or hypotonic medium).

\section{Analysis of live imaging data}

\section{Pre-processing of timelapses:}

All timelapses were smoothed in time using a Simoncelli smoothing filter [52]. Prior to smoothing, any timelapses that showed noticeable jitter or drift due to thermal gradients were processed through a jitter correction algorithm using discrete Fourier transform (DFT) registration and an upsampling factor of 100 (using the "dftregistration" code package from [53]). For timelapses where drift was inconsistent and unable to be fully corrected with the DFT method, an additional correction using intensity-based image registration (via the MATLAB functions "imregconfig" and "imregister") was applied using the average image of the DFT corrected timelapse as the fixed image.

Shape analysis and identification of bulk and boundary regions:

We used shape analysis to identify cell boundaries and subsequently to be able to compare actin dynamics within different regions of astrocytes. Using a snake algorithm from our previous work [16, 29, 30], we analyzed the average fluorescent image of each cell to generate the "true boundary" (see Figure 1A-B). Prior to shape analysis, images were smoothed ("imgaussfilt" in MATLAB with a standard deviation of 1.0) and subsequently sharpened ("imsharpen" in MATLAB with a radius of 2.5 and amount of 1.0). Alpha and beta parameters for the snake algorithm (tension and rigidity, respectively) were 0.20 and 0.25 , respectively. We found that this true boundary tended to capture the most prominent morphological features of the cell but often excluded regions that were only transiently dynamic. To mitigate this challenge, we eroded or dilated the true boundary using a spherical structural element with a radius of 10 pixels. By subtracting the eroded cell region from the dilated cell region, we identified a boundary region. We considered the eroded cell mask to be the bulk region.

\section{Optical flow analysis of actin dynamics:}

Optical flow analysis similar to that used in [19] was performed on smoothed and jitter-corrected timelapses in Python 3.8. The optical flow weight matrix was a 19x19 pixel Gaussian with a standard deviation of 3 pixels $(1.1 \mu \mathrm{m})$ for all timelapses taken at UMD. For a small subset of 
HTlc timelapses taken at the CNR, the optical flow weight matrix was a $41 \times 41$ pixel Gaussian with a standard deviation of 6.75 pixels $(1.1 \mu \mathrm{m})$. The truncation value for the Gaussian kernel was consistently set to 3 . Because actin dynamics in astrocytes is less prominent than in migrating cells usually studied by our lab $[15,19]$, we only considered optical flow vectors with the highest $1 \%$ of magnitude within the dilated cell boundary that also passed our reliability criteria (at least 33\% of the mean reliability value for that timelapse). These strict criteria ensured that we were only considering dynamic actin events that are large and reliable compared to background noise.

\section{Segmentation of active regions ("hotspots") of actin dynamics:}

To identify regions with the most actin activity, we further analyzed our thresholded data by eroding and dilating in the $\mathrm{x}, \mathrm{y}$, and time dimensions using an elongated cube with dimensions of $[3,3,2]$. To ensure that we were not including regions of low activity, we set an event probability threshold of 0.10 , meaning that a region had to be active at least $10 \%$ of the time to be considered a "hotspot". Furthermore, we imposed a size constraint of 20 pixels (including the time dimension) to ensure that any active regions did not just barely meet the volume require of $3 \times 3 \times 2$. Since the dynamics within HTlc cells are on a smaller scale, the microdomain analysis did not reveal any regions that were clearly active.

\section{Analysis of STED images of fixed astrocytes}

\section{Laplacian of Gaussian (LoG) filtering to segment actin:}

To characterize the organization of the actin meshwork in STED images of fixed cells, as shown in Figure 4, we performed several image processing techniques using custom-written algorithms in MATLAB (Mathworks). Prior to processing, all STED images are adjusted using a contrastlimited adaptive histogram equalization ("adjusthisteq" function in MATLAB's proprietary API (https://www.mathworks.com/help/images/adaptive-histogram-equalization.html). The resolution of the STED images is $16.18 \mathrm{pix} / \mathrm{nm}$. Next, each image is convolved with an anisotropic, rotating Laplacian of Gaussian (LoG) kernel. The exact kernel parameters were determined through trial and error to approximately match the cylindrical shape of actin as visualized by Phalloidin-488 staining. The number of angles through which the LoG kernel is rotated was chosen to balance computational time and segmentation accuracy. For each pixel, the best match angle is chosen via the maximum value resulting from convolving that pixel with all rotations of the filter, and a threshold is applied to ensure a high-quality match. This processing results in a filtered image that highlights both the actin organization and the angle associated with each filament extracted from kernel (see Figure 5A1). The angles associated with the extracted actin are defined in terms of the Cartesian coordinate system, which is not biologically meaningful. To establish the angle at which the actin organizes relative to the cellular boundary, we manually draw a boundary for each cell. The number of pixels per boundary varies on a cell-to-cell basis. For each pixel, we derive the angle of that pixel's actin relative to the closest boundary point. This transformation generates angles in the range of 0 to $\pi / 2$. We then apply an additional threshold at an arbitrary value to only segment the longest actin (Figure 5A2); doing so greatly reduces the noise in our processed data. We analyzed at least 15 individual cells per nanotopographic surface, and the morphologies of the imaged areas varied from cell to cell. Thus, the boundaries are generated to normalize the distributions of the relative angles across the data sets. We noticed that for the STED images taken of astrocytes grown on HTlc, there is more total boundary per unit-area. 
Thus, the boundaries from which the relative angles are calculated are drawn to coincide with only the leading edge of the cell (see Figure 5B for representative examples).

Cluster analysis of relative angles:

To understand how astrocytic actin organization differs when the cells are grown on different nanotopographies, we use a hierarchical cluster analysis from MATLAB's proprietary API (https://www.mathworks.com/help/stats/cluster-analysis.html). From the relative angle distributions across both PDL and HTlc (22 individual cells and 15 individual cells, respectively), we group the distributions into "parallel" (angles between 0 and $\pi / 6$ ) and "perpendicular" (angles between $\pi / 3$ and $\pi / 2$ ), as shown in Figure 5B. As inputs to the clustering algorithm, we use the fractions of "parallel" and "perpendicular" actin of the 37 individual cells but do not include information about the corresponding nanotopographic surface. We then use the "pdist," "linkage," "cophenet," and "linkage" functions (while optimizing the cophenetic coefficient of our 'distance' and 'linkage' parameters) to generate predictive clusters of the input data.

\section{Data representation and statistics}

All data were plotted and all statistical tests were performed in MATLAB 2021a. For data showing differences between control and triggering or between boundary and bulk regions, paired t-tests were used ("ttest" function in MATLAB). For data comparing PDL to HTlc, unpaired two-sample t-tests were used ("ttest2" function in MATLAB). When analyzing optical flow prevalence vs. distance, ANOVA tests were used: repeated measures ANOVA was used for paired data in Figure 1 ("fitrm" and "ranova" functions in MATLAB), and two-way ANOVA was used to compare PDL and HTlc in Figure 3 ("anovan" function in MATLAB). In both cases, Tukey's multiple comparisons test ("multcompare" function in MATLAB) was used as the post test. All errorbars indicate $95 \%$ confidence interval, and the significance level was set at $\alpha=0.05$. Adobe Illustrator (version 25.0.1) was used for final assembly of all figures.

\section{$\underline{\text { References }}$}

1. Verkhratsky, A. and M. Nedergaard, Physiology of Astroglia. Physiol Rev, 2017. 98(1):

2. Rose, C.R. and A. Verkhratsky, Principles of sodium homeostasis and sodium signalling in astroglia. Glia, 2016. 64(10): p. 1611-1627.

3. Benfenati, V. and S. Ferroni, Water transport between CNS compartments: Functional and molecular interactions between aquaporins and ion channels. Neuroscience, 2010. 168(4): p. 926-940.

4. Santello, M., N. Toni, and A. Volterra, Astrocyte function from information processing to cognition and cognitive impairment. Nat Neurosci, 2019. 22(2): p. 154-166.

5. Farhy-Tselnicker, I., et al., Activity-dependent modulation of synapse-regulating genes in astrocytes. Elife, 2021. 10: p. e70514.

6. Bellot-Saez, A., et al., Astrocytic modulation of neuronal excitability through $K+$ spatial buffering. Neurosci Biobehav Rev, 2017. 77: p. 87-97.

7. Scemes, E. and D.C. Spray, Extracellular $K^{+}$and astrocyte signaling via connexin and pannexin channels. Neurochem Res, 2012. 37(11): p. 2310-2306. 
639 8. Spray, D.C., Molecular physiology of gap junction channels. Clinical and experimental

671

672

673

674

675

676

677

678

679

680

681

682 pharmacology and physiology, 1996. 23(12): p. 1038-1040.

9. Scemes, E. and D.C. Spray, Extracellular $K+$ and astrocyte signaling via connexin and pannexin channels. Neurochemical research, 2012. 37(11): p. 2310-2316.

10. Nicchia, G.P., et al., Actin cytoskeleton remodeling governs aquaporin-4 localization in astrocytes. Glia, 2008. 56(16): p. 1755-1766.

11. Benfenati, V., et al., Functional down-regulation of volume-regulated anion channels in AQP4 knockdown cultured rat cortical astrocytes. Journal of Neurochemistry, 2007. 100(1): p. 87-104.

12. Nicchia, G.P., et al., Different pattern of aquaporin-4 expression in extensor digitorum longus and soleus during early development. Muscle Nerve, 2007. 35(5): p. 625-631.

13. Fritzsche, M., et al., Cytoskeletal actin dynamics shape a ramifying actin network underpinning immunological synapse formation. Sci Adv, 2017. 3(6): p. e1603032.

14. Vicker, M.G., F-actin assembly in Dictyostelium cell locomotion and shape oscillations propagates as a self-organized reaction-diffusion wave. FEBS Letters, 2002. 510(1-2): p. 5-9.

15. Sun, X., et al., Asymmetric nanotopography biases cytoskeletal dynamics and promotes unidirectional cell guidance. Proc Natl Acad Sci U S A, 2015. 112(41): p. 12557-12562.

16. Driscoll, M.K., et al., Cellular contact guidance through dynamic sensing of nanotopography. ACS Nano, 2014. 8(4): p. 3546-3555.

17. Edwards, M., et al., Insight from the maximal activation of the signal transduction excitable network in Dictyostelium discoideum. Proc Natl Acad Sci U S A, 2018. 115(16): p. E3722-E3730.

18. Miao, Y., et al., Wave patterns organize cellular protrusions and control cortical dynamics. Mol Syst Biol, 2019. 15(3): p. e8585.

19. Lee, R., et al., Quantifying topography-guided actin dynamics across scales using optical flow. Mol Biol Cell, 2020. mbcE19110614.

20. Roy, S., Waves, rings, and trails: The scenic landscape of axonal actin. J Cell Biol, 2016. 212(2): p. 131-134.

21. Del Signore, S.J., et al., An autoinhibitory clamp of actin assembly constrains and directs synaptic endocytosis. Elife, 2021. 10: p. e69597.

22. Li, X., et al., Excitable networks controlling cell migration during development and disease. Semin Cell Dev Biol, 2020. 100: p. 133-142.

23. Pal, D.S., et al., The excitable signal transduction networks: movers and shapers of eukaryotic cell migration. Int J Dev Biol, 2019. 63(8-9-10): p. 407-416.

24. Azatov, M., et al., Topography on a subcellular scale modulates cellular adhesions and actin stress fiber dynamics in tumor associated fibroblasts. Phys Biol, 2017. 14(6): p. 065003.

25. Ketchum, C.M., et al., Subcellular topography modulates actin dynamics and signaling in B-cells. Mol Biol Cell, 2018. 29(13): p. 1732-1742.

26. Kumari, S., et al., Cytoskeletal tension actively sustains the migratory T-cell synaptic contact. EMBO J, 2020. 39(5): p. e102783.

27. Bernier, L.-P., et al., Nanoscale surveillance of the brain by microglia via cAMPregulated filopodia. Cell Rep, 2019. 27(10): p. 2895-2908.e4. 
28. Lucas, B.D. and T. Kanade. An iterative image registration technique with an application to stereo vision. in Proceedings of the 7th International Joint Conference on Artificial Intelligence. 1981. San Francisco, CA, USA: Morgan Kaufmann Publishers Inc.

29. Driscoll, M.K., et al., Spatiotemporal relationships between the cell shape and the actomyosin cortex of periodically protruding cells. Cytoskeleton (Hoboken), 2015 72(6): p. 268-281.

30. Driscoll, M.K., et al., Cell shape dynamics: from waves to migration. PLoS Comput Biol, 2012. 8(3): p. e1002392.

31. Posati, T., et al., A nanoscale interface promoting molecular and functional differentiation of neural cells. Sci Rep, 2016. 6: p. 31226.

32. Mola, M.G., et al., Cell volume regulation mechanisms in differentiated astrocytes. Submitted, 2021.

33. Campanello, L., et al., Signaling through polymerization and degradation: Analysis and simulations of T cell activation mediated by Bcl10. PLoS Comput Biol, 2021. 17(5): $\mathrm{p}$. e1007986.

34. Schiweck, J., B.J. Eickholt, and K. Murk, Important shapeshifter: Mechanisms allowing astrocytes to respond to the changing nervous system during development, injury and disease. Front Cell Neurosci, 2018. 12: p. 261.

35. Schiweck, J., et al., Drebrin controls scar formation and astrocyte reactivity upon traumatic brain injury by regulating membrane trafficking. Nat Commun, 2021. 12: p. 1490.

36. Ferroni, S., et al., Two distinct inwardly rectifying conductances are expressed in long term dibutyryl-cyclic-AMP treated rat cultured cortical astrocytes. FEBS Lett, 1995. 367(3): p. 319-325.

37. Benfenati, V., et al., Guanosine promotes the up-regulation of inward rectifier potassium current mediated by Kir4.1 in cultured rat cortical astrocytes. J Neurochem, 2006. 98(2): p. 430-445.

38. McCarthy, K.D. and J. de Vellis, Preparation of separate astroglial and oligodendroglial cell cultures from rat cerebral tissue. J Cell Biol, 1980. 85(3): p. 890-902.

39. Bernardinelli, Y., et al., Activity-dependent structural plasticity of perisynaptic astrocytic domains promotes excitatory synapse stability. Curr Biol, 2014. 24(15): p. 1679-1688.

40. Hirrlinger, J., S. Hülsmann, and F. Kirchhoff, Astroglial processes show spontaneous motility at active synaptic terminals in situ. Eur J Neurosci, 2004. 20(8): p. 2235-2239.

41. Ferroni, S., et al., Characterization of an inwardly rectifying chloride conductance expressed by cultured rat cortical astrocytes. Glia, 1997. 21(2): p. 217-227.

42. Nicchia, G.P., et al., Aquaporin-4-containing astrocytes sustain a temperature- and mercury-insensitive swelling in vitro. Glia, 2000. 31(1): p. 29-38.

43. Muñoz-Lasso, D.C., et al., Much more than a scaffold: Cytoskeletal proteins in neurological disorders. Cells, 2020. 9(2): p. 358.

44. Tobe, B.T.D., et al., Probing the lithium-response pathway in hiPSCs implicates the phosphoregulatory set-point for a cytoskeletal modulator in bipolar pathogenesis. Proc Natl Acad Sci U S A, 2017. 114(22): p. E4462-E4471.

45. Benfenati, V., et al., A silk platform that enables electrophysiology and targeted drug delivery in brain astroglial cells. Biomaterials, 2010. 31(31): p. 7883-7891. 
760

761

762

763

764

765

766

767

768

769

770

771

772

46. Saracino, E., et al., A Glial-Silicon Nanowire Electrode Junction Enabling Differentiation and Noninvasive Recording of Slow Oscillations from Primary Astrocytes. Adv Biosyst, 2020. 4(4): p. e1900264.

47. Benfenati, V. and S. Ferroni, Microdynamics of Water and Ion Homeostasis in the Brain, in Homeostatic Control of Brain Function. 2015, Oxford University Press.

48. Benfenati, V., et al., Photostimulation of whole-cell conductance in primary rat neocortical astrocytes mediated by organic semiconducting thin films. Adv Healthc Mater, 2014. 3(3): p. 392-399.

49. Borrachero-Conejo, A.I., et al., Stimulation of water and calcium dynamics in astrocytes with pulsed infrared light. Faseb J, 2020. 34(5): p. 6539-6553.

50. De Bellis, M., et al., Translational readthrough generates new astrocyte AQP4 isoforms that modulate supramolecular clustering, glial endfeet localization, and water transport. Glia, 2017. 65(5): p. 790-803.

51. de Bellis, M., et al., Orthogonal arrays of particle assembly are essential for normal aquaporin-4 expression level in the brain. Glia, 2021. 69(2): p. 473-488.

52. Simoncelli, E.P., Design of multi-dimensional derivative filters. Proceedings of $1 \mathrm{st}$ International Conference on Image Processing, 1994. 1: p. 790-794.

53. Guizar-Sicairos, M., S.T. Thurman, and J.R. Fienup, Efficient subpixel image registration algorithms. Opt Lett, 2008. 33: p. 156-158.

\section{Acknowledgements}

The authors would like to thank the University of Maryland Imaging Incubator Core Facility for providing and maintaining the systems used in collecting images for this work. They also thank Q. Yang and L. J. Campanello for helpful discussions related to analysis.

Funding: This work was supported by the following grants:

US Air Force Office of Scientific Research MURI grant FA9550-16-1-0052 to WL US Air Force Office of Scientific Research grant FA9550-19-1-0370 (AstroDyn) to WL VB GPN KMO US Air Force Office of Scientific Research FA9550-16-1-0502 (AstroMat) to VB US Air Force Office of Scientific Research FA9550-17-1-0502 (AstroNir) to VB US Air Force Office of Scientific Research FA9550-18-1-0255 (3D Neuroglia) to VB COMBINE fellowships to NJM SP

\section{Author contributions}

Conceptualization: VB WL

Surface preparation: ES TP

Experiments: KMO ES BB MGM

Analysis: KMO NJM SP

Interpretation: KMO ES BB NJM MGM GPN VB WL

Supervision: GPN VB WL

Writing - original draft: KMO ES BB NJM GPN VB WL

Writing — review \& editing: KMO ES BB NJM MGM SP TP RZ GPN VB WL

Competing interests: The authors have no competing interests to declare. 
bioRxiv preprint doi: https://doi.org/10.1101/2021.09.13.460152; this version posted September 13, 2021. The copyright holder for this preprint (which was not certified by peer review) is the author/funder. All rights reserved. No reuse allowed without permission.

773 Data and materials availability: All data and code will be made available in a repository 774 located in the Losert laboratory's github page.

775 
A

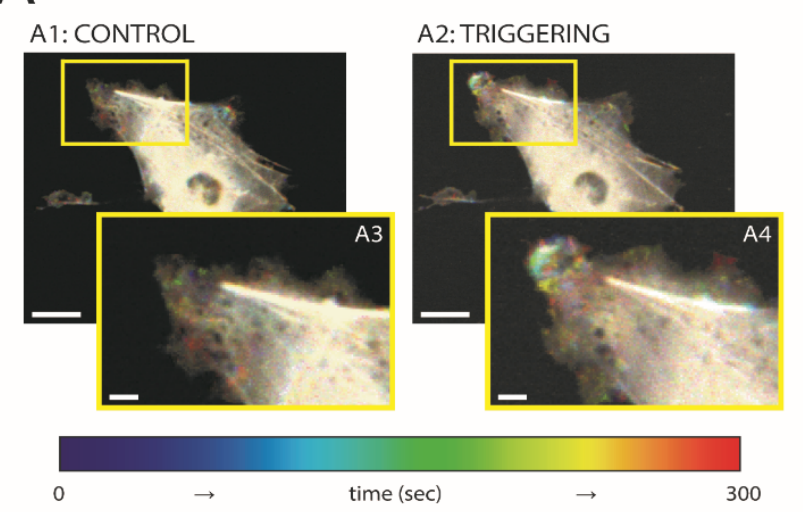

$\mathrm{C}_{\mathrm{c}}$

boundary region (B) bulk region distance (d) to nearest boundary point

$\mathrm{D}$

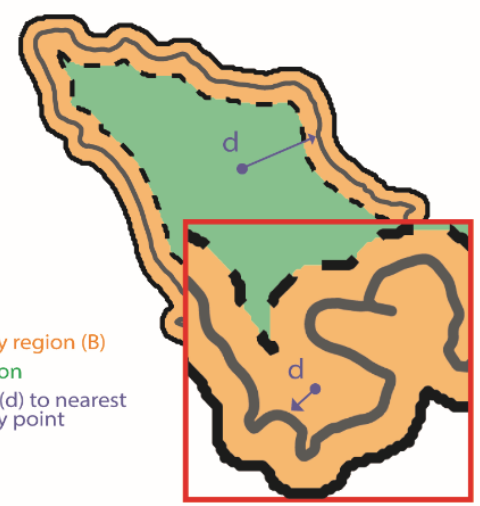

D1

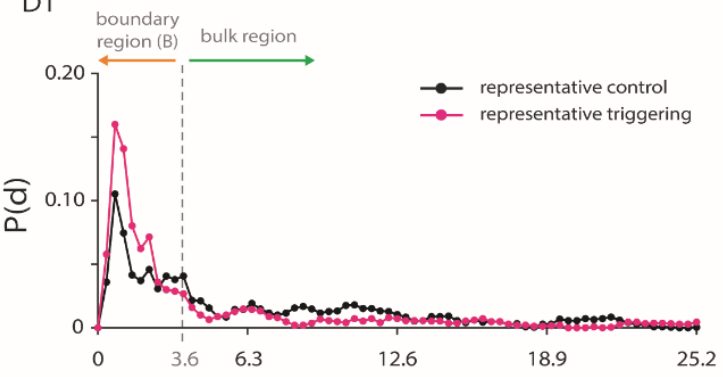

$\mathrm{d}(\mu \mathrm{m})$

B

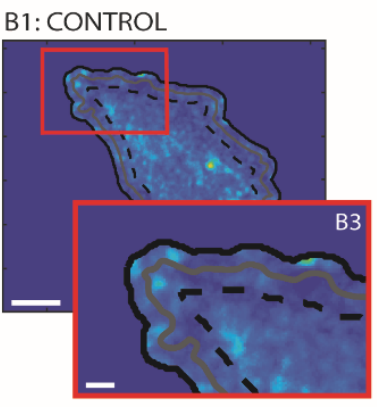

boundary legend:

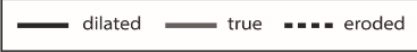

C2: Strength

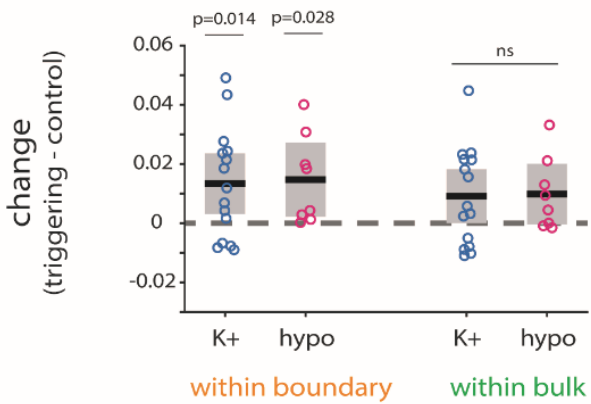

B2: TRIGGERING

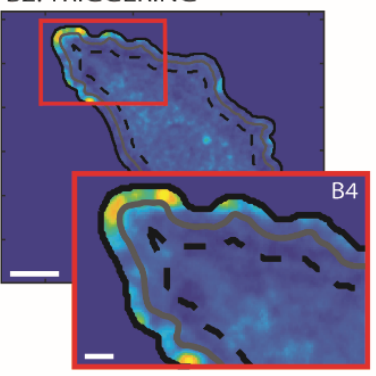

avg. magnitude:

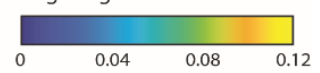

C3: Relative fraction

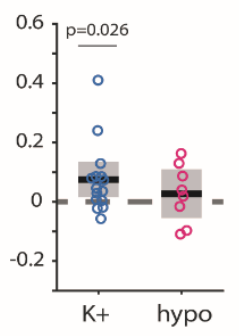

778

779

780

781

782

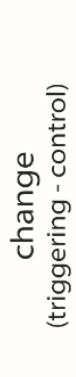

D2: $P(d$ within $B)$
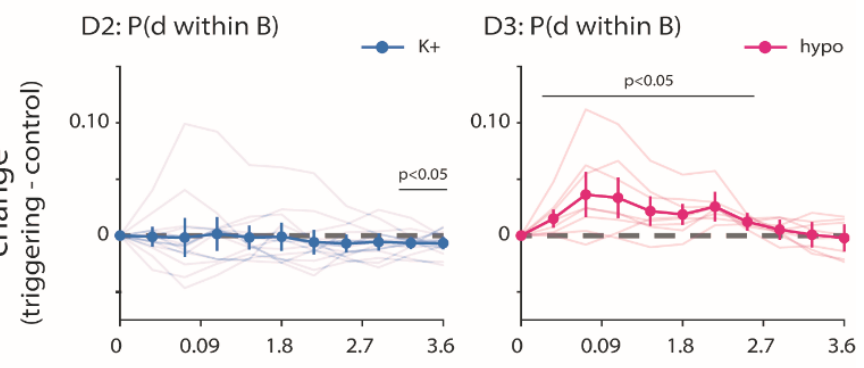

d within $B(\mu \mathrm{m})$

Figure 1: Optical flow analysis reveals actin dynamics in primary astrocytes.

A) Actin-GFP timelapse for representative cell, control (A1, inset A3) and triggering (A2, inset A4) conditions. Colorbar indicates time. B) Optical flow analysis for representative cell from A, control (B1, inset B3) and triggering (B2, inset B4) conditions. Colorbar indicates average optical flow magnitude. C) Characterizing actin dynamics. C1) Shape analysis [16, 29, 30] 
785 identifies true boundary (solid grey line). Boundary region (orange) is created by dilating (solid 786 black line) and eroding (dashed black line) true boundary by 10 pixels and is distinct from bulk 787 region (green). C2) Strength of actin dynamics for control vs. $K+(\mathrm{p}=0.014$ for boundary, $788 \mathrm{p}=0.050$ for bulk) and for control vs. hypo ( $\mathrm{p}=0.028$ for boundary, $\mathrm{p}=0.057$ for bulk). 789 C3) Relative fraction of actin dynamics within boundary for control vs. $\mathrm{K}+(\mathrm{p}=0.026)$ and for 790 control vs. hypo ( $\mathrm{p}=0.466)$. D) Distance dependence of actin dynamics. D1) Probability $P(d)$ vs. 791 distance from boundary $d$ for representative data shown in B. D2-D3) Probability within 792 boundary region $P(d$ within $B)$ vs. distance from true boundary within boundary region $d$ within $793 B$ for all cells for control vs. $\mathrm{K}+(\mathrm{D} 2 ; \mathrm{p}=0.019$ at $3.24 \mu \mathrm{m}, \mathrm{p}=0.021$ at $3.6 \mu \mathrm{m})$ and for control vs.

794 hypo $(\mathrm{D} 3 ; \mathrm{p}=0.033, \mathrm{p}=0.032, \mathrm{p}=0.030, \mathrm{p}=0.036, \mathrm{p}=0.025, \mathrm{p}=0.001, \mathrm{p}<0.001$ at $0.36-2.52 \mu \mathrm{m})$. P795 values determined by paired t-test $(\mathrm{C})$ or by repeated measures ANOVA (D). $\mathrm{n}=15$ paired cells 796 for control and $\mathrm{K}+$, and $\mathrm{n}=8$ paired cells for control and hypo. Scalebars indicate $20 \mu \mathrm{m}$ (A1-A2, 797 B1-B2) or $5 \mu \mathrm{m}$ (A3-A4, B3-B4). Error bars/boxes indicate 95\% confidence intervals. Solid 798 black lines and opaque circles indicate mean. Transparent lines in D2-D3 represent individual 799 distance curves. "Control" indicates cells imaged in standard external medium; "triggering" 800 indicates cells imaged in isotonic high potassium solution ("K+") or hypotonic solution 801 ("hypo"). 
A

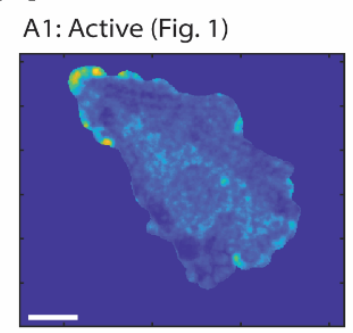

avg. probability:

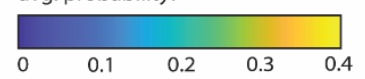

A2: Persistent
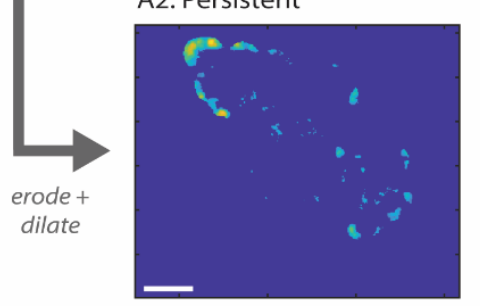

C

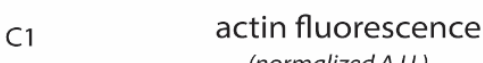
(normalized A.U.)

CONTROL

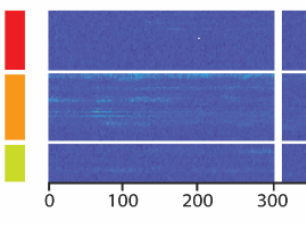

B
804

805

806

807

808

809

810

811

814 812 boundary, $\mathrm{p}=0.527$ for bulk). B2) Relative strength of actin dynamics for control vs. K+ $813(\mathrm{p}=0.028)$ and for control vs. hypo $(\mathrm{p}=0.087)$. B3) Average persistence of actin dynamics for

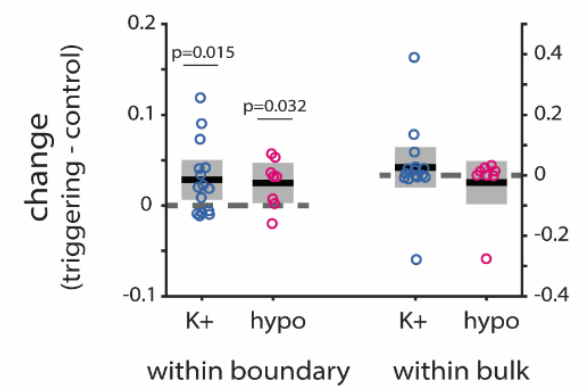

B2: Relative strength

B3: Persistence
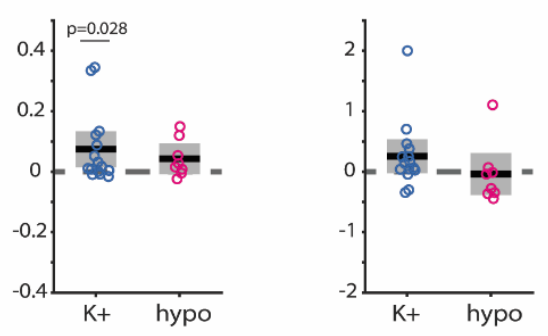

A3: Segmented
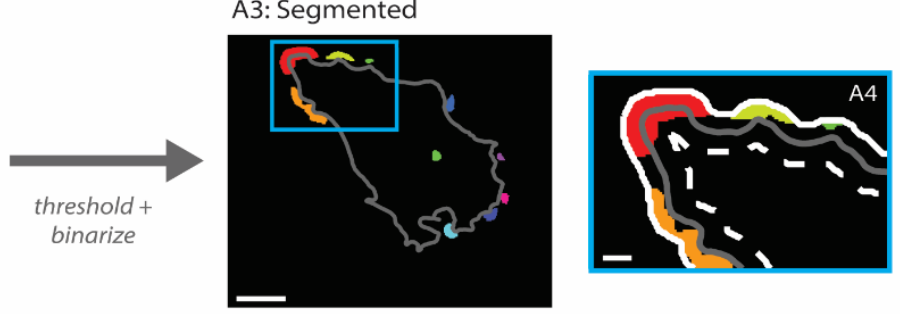

boundary legend:

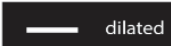

$\begin{array}{ll} & \text { true } \\ \ldots-.- & \text { eroded }\end{array}$

C2 $\begin{gathered}\text { actin dynamics } \\ \text { (optical flow magnitude) }\end{gathered}$

CONTROL

TRIGGERING

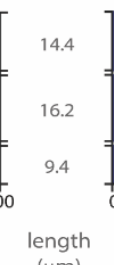

$(\mu \mathrm{m})$
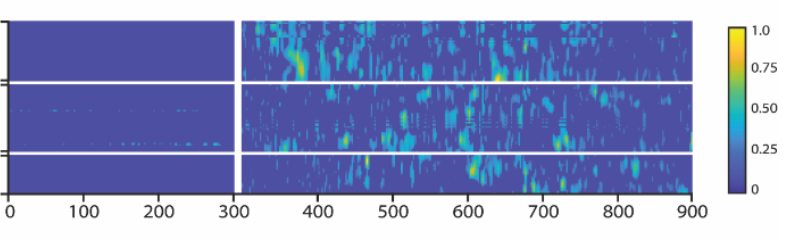

time (sec)

\section{Figure 2: Persistence analysis of actin dynamics reveal "hotspots" of actin activity.}

A) Identification of "hotspot" regions. Optical flow results (A1; from Figure 1) are eroded and dilated in space and in time (A2) to identify the most persistently active regions. Regions that meet criteria are binarized and segmented (A3, inset A4) for comparison between cells and conditions. B) Characterization of hotspot dynamics. B1) Average strength of actin dynamics for control vs. $\mathrm{K}+(\mathrm{p}=0.015$ for boundary, $\mathrm{p}=0.464$ for bulk) and for control vs. hypo ( $\mathrm{p}=0.032$ for control vs. $\mathrm{K}+(\mathrm{p}=0.095)$ and for control vs. hypo $(\mathrm{p}=0.831)$. All $\mathrm{p}$-values determined by paired 
815 t-test. Solid black lines indicate the mean. Grey regions indicate $95 \%$ confidence interval.

816 C) Kymographs of normalized actin fluorescence $(\mathrm{C} 1)$ and of optical flow magnitude (C2) for

817 three largest hotspot regions from A3-A4. Grey labels between kymographs indicate lengths of

818 regions. Colorbar indicates normalized fluorescence (C1) and optical flow magnitude (C2). n=15

819 paired cells for control and $\mathrm{K}+$, and $\mathrm{n}=8$ paired cells for control and hypo. 

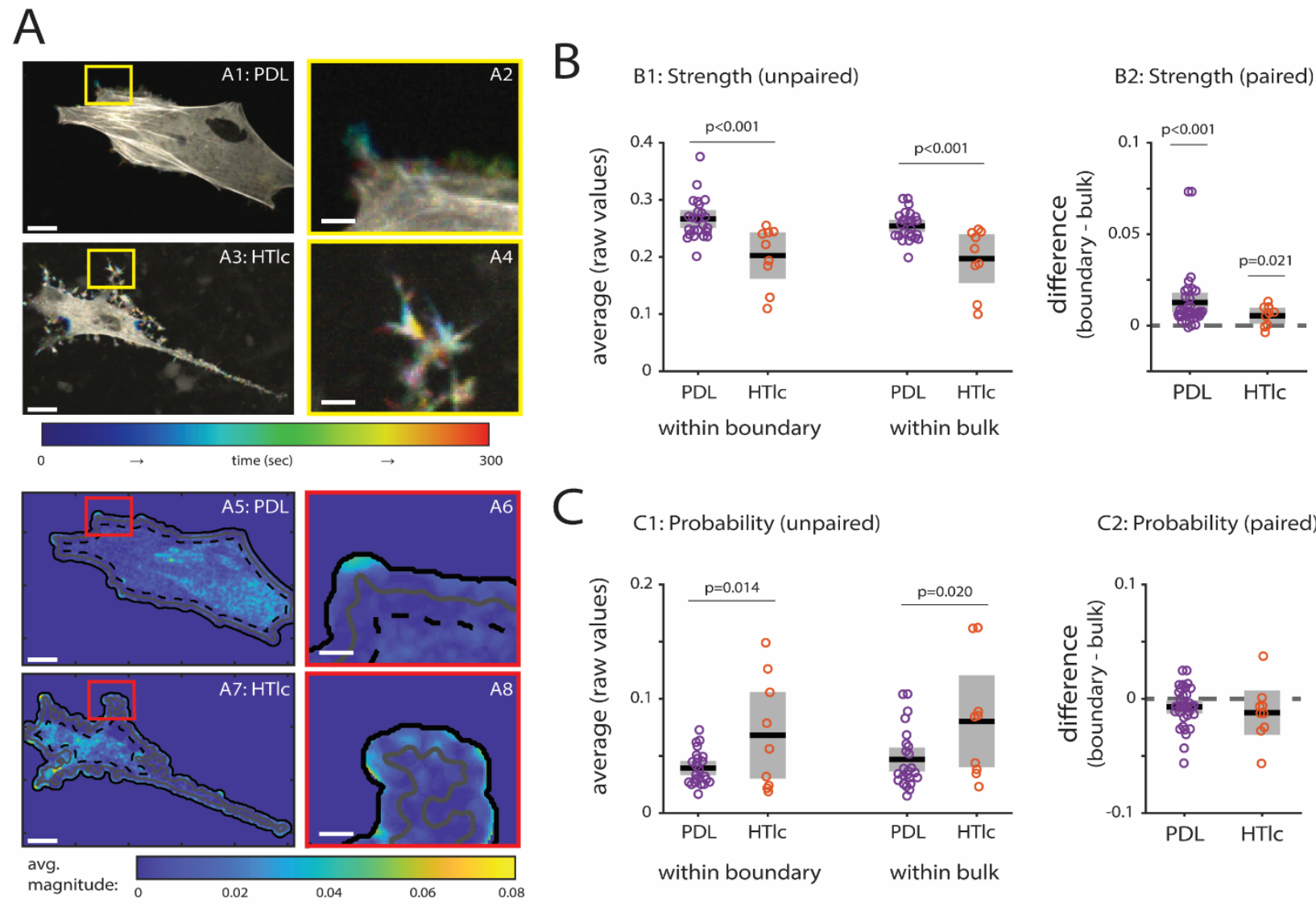

C2: Probability (paired)

D
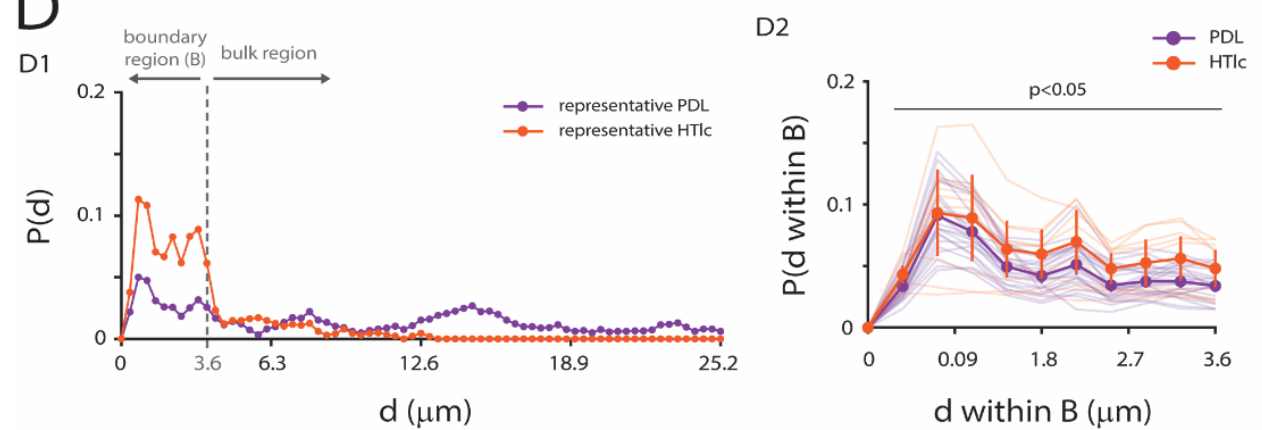

d within $B(\mu \mathrm{m})$

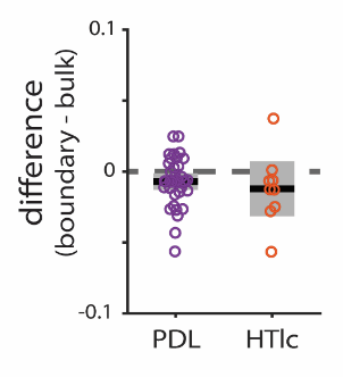

Figure 3: Actin dynamics differs substantially in undifferentiated astrocytes grown on PDL

825 compared to differentiated astrocytes grown on HTlc.

826 A) Actin-GFP timelapse for representative cells grown on PDL-coated glass ("PDL"; A1, inset

827 A2) and HTlc films ("HTlc"; A3, inset A4). Colorbar indicates time. Optical flow analysis for 828 representative cells (PDL in A5, inset A6; HTlc in A7, inset A8). Colorbar indicates average 829 optical flow magnitude. B) Strength of actin dynamics. B1) Strength of actin dynamics within 
830 boundary $(\mathrm{p}<0.001)$ and within bulk $(\mathrm{p}<0.001)$ for PDL vs. HTlc cells. B2) Strength of actin

831 dynamics in boundary vs. bulk for PDL $(p<0.001)$ and for HTlc $(p=0.021)$ cells. C) Probability

832 of actin dynamics. C1) Probability of actin dynamics within boundary $(p=0.014)$ and within bulk

$833(\mathrm{p}=0.020)$ for PDL vs. HTlc cells. C2) Probability of actin dynamics in boundary vs. bulk for

834 PDL $(p=0.072)$ and for HTlc $(p=0.184)$ cells. D) Distance dependence of actin dynamics.

835 D1) Probability $P(d)$ vs. distance from boundary $d$ for representative data shown in A5-A8.

836 D2) Probability within boundary region $P(d$ within $B)$ vs. distance from true boundary within

837 boundary region $d$ within $B$ for all PDL and HTlc cells $(\mathrm{p}<0.001$ at $0.36-3.24 \mu \mathrm{m}, \mathrm{p}=0.01$ at

$8383.6 \mu \mathrm{m})$. D3) Relative fraction of actin dynamics within boundary $(\mathrm{p}=0.287$ for PDL, $\mathrm{p}=0.058$

839 for HTlc). P-values determined by unpaired t-test (B1, C1), paired t-test (B2, C2, D3), or two-

840 way ANOVA (D2). $n=24$ cells for PDL, and $n=9$ cells for HTlc. Scalebars indicate $20 \mu \mathrm{m}(\mathrm{A} 1$,

$841 \mathrm{~A} 3, \mathrm{~A} 5, \mathrm{~A} 7)$ or $5 \mu \mathrm{m}(\mathrm{A} 2, \mathrm{~A} 4, \mathrm{~A} 6, \mathrm{~A} 8)$. Error bars/boxes indicate $95 \%$ confidence intervals.

842 Solid black lines and opaque circles indicate mean. Transparent lines in D2 represent individual

843 distance curves. 

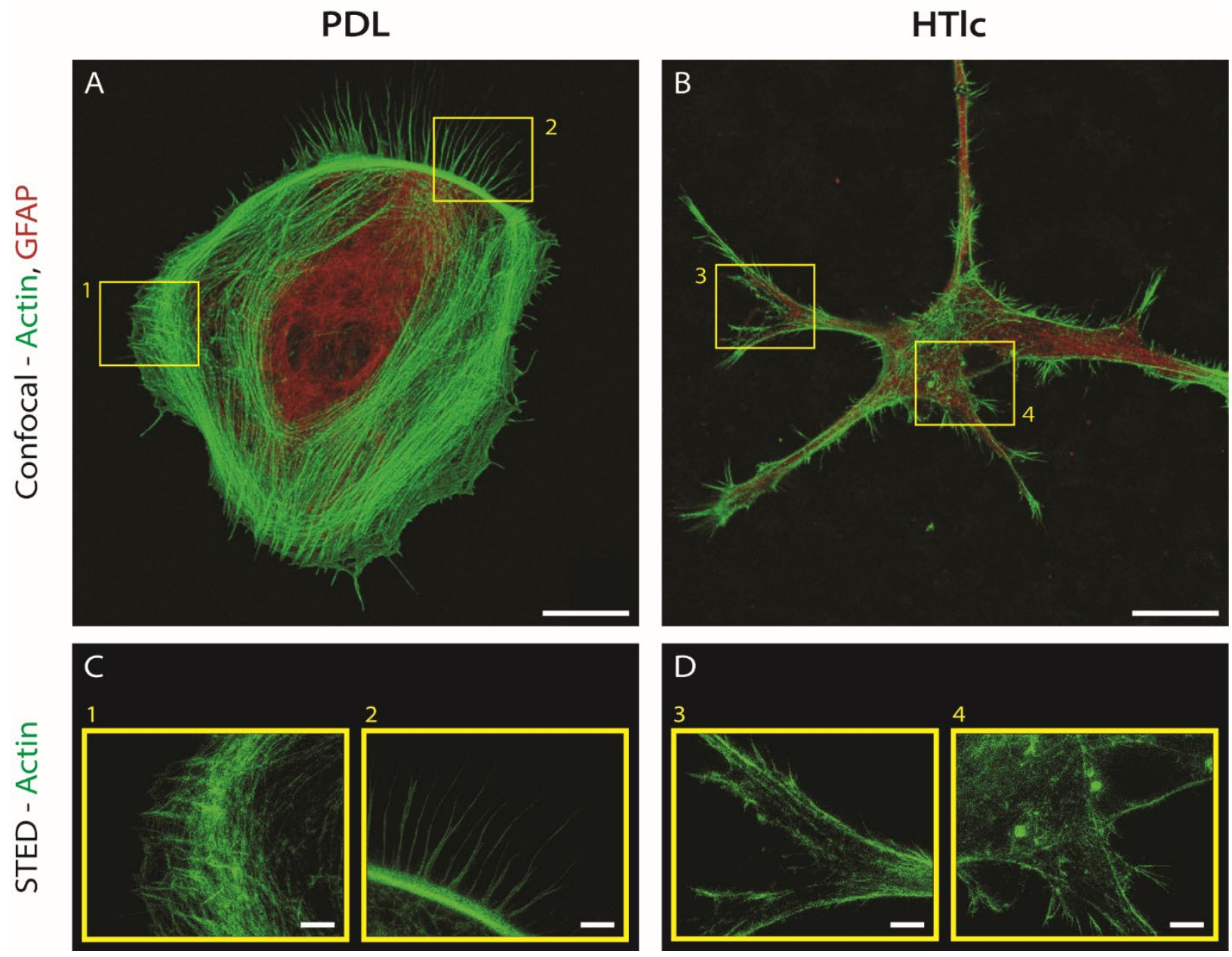

Figure 4: STED microscopy visualizes nanoscale actin structure within primary astrocytes grown on PDL and on HTIc. 
A

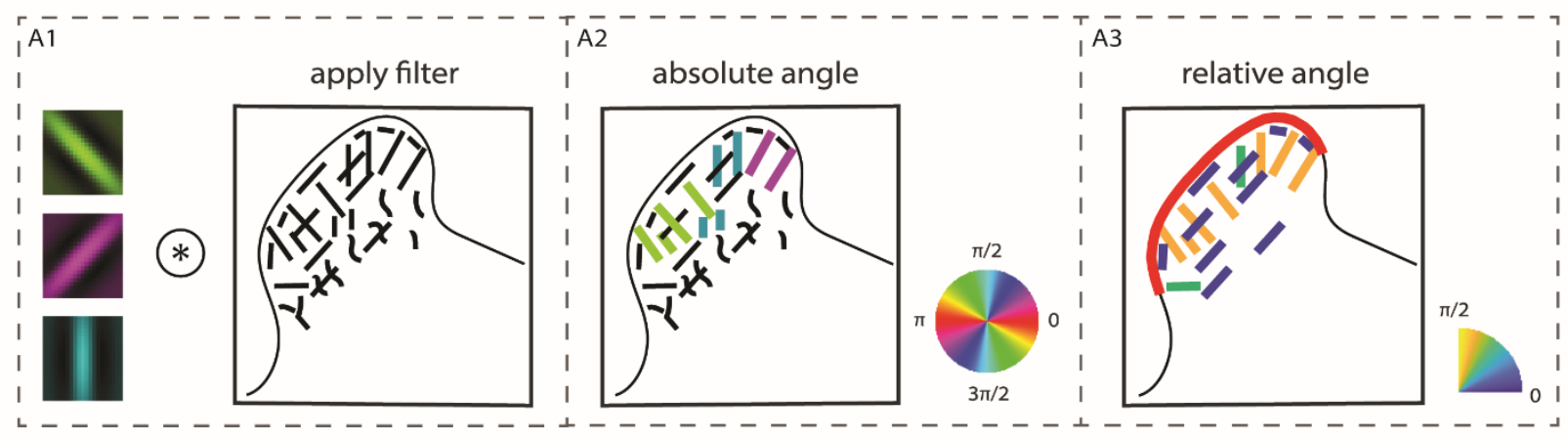

B

856

857

858

859

860

861

862

863

864

865

866
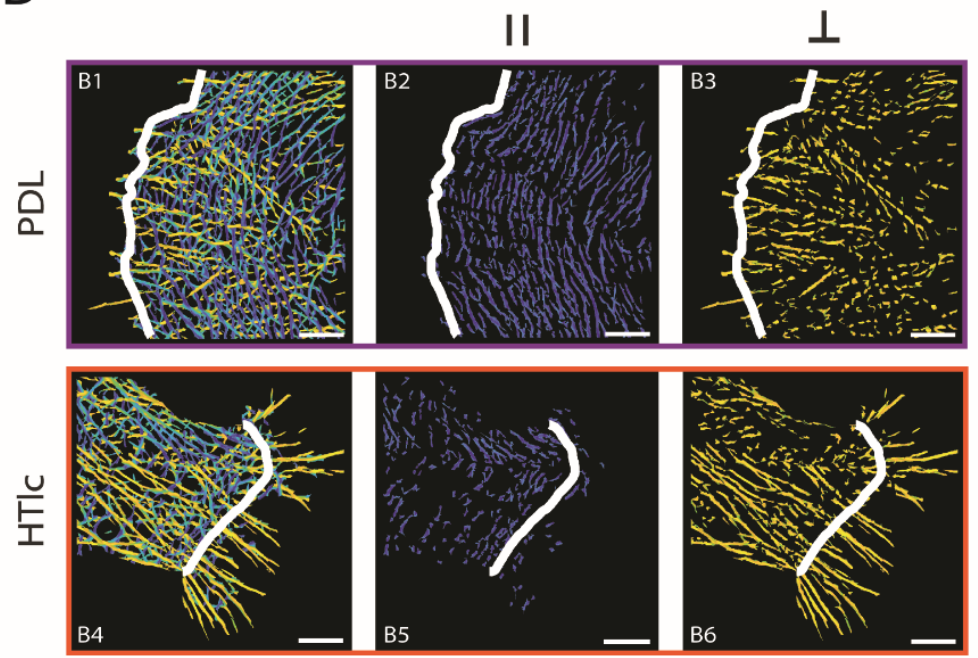

II

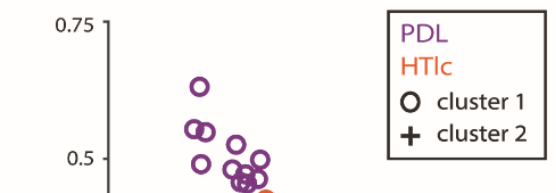

filament orientation fraction

Figure 5: Filtering STED images with a rotating, anisotropic LoG kernel reveals differences in nanoscale actin structure for astrocytes grown on PDL versus on HTlc.

A) Extraction of relative angle information from actin filaments. A1) STED images are convolved with a rotating, anisotropic LoG kernel. A2) Filtering extracts actin bundles and a best match angle that is relative to the MATLAB frame ("absolute angle") with a range of $[0,2 \pi]$ (shown as full colorwheel). A3) A leading edge boundary (red) is drawn manually and is used to transform actin angles to be relative to the closes boundary point ("relative angle") with a range of $[0, \pi / 2]$ (shown as a quarter colorwheel). B) Output from A for representative cells (PDL in B1-B3, HTlc in B4-B6). All relative angles, parallel angles only (range of $[0, \pi / 6]$ ), and 
867 perpendicular angles only (range of $[\pi / 3, \pi / 2]$ ) for representative PDL (B1, B2, B3, respectively)

868 and HTlc (B4, B5, B6, respectively) cells. Solid white lines indicate leading edge boundary.

869 Scalebars indicate $2.5 \mu \mathrm{m}$. C) Parallel (y-axis) vs. perpendicular (x-axis) fractions of actin

870 detected within PDL (purple) and HTlc (orange) astrocytes. A hierarchical cluster analysis

871 reveals two distinct clusters (cluster 1: open circles, cluster 2: crosses). Arrows indicate

872 representative cells. $n=22$ cells for PDL, and $n=15$ cells for HTlc.

873 
874

\begin{tabular}{|c|c|c|c|}
\hline & Parameter & Actin & GFAP \\
\hline \multirow[t]{2}{*}{ Confocal } & $\begin{array}{c}\text { WLL (488 nm) } \\
\text { intensity, } \%\end{array}$ & 10 & 10 \\
\hline & Gain & 25 & 25 \\
\hline \multirow[t]{3}{*}{ STED } & $\begin{array}{l}\text { WLL (488 nm) } \\
\text { intensity, \% }\end{array}$ & 40 & \\
\hline & $\begin{array}{c}\text { STED (592 nm) } \\
\text { intensity, } \%\end{array}$ & 97 & \\
\hline & Gain & 200 & \\
\hline
\end{tabular}

875

876 Table 1: Imaging parameters. Parameters used for STED and confocal imaging of fixed 877 astrocytes on PDL and HTlc.

878 